\section{Tendência da mortalidade neonatal na cidade de Salvador (Bahia-Brasil), 1996-2012}

\section{Neonatal mortality trends in the city of Salvador (Bahia-Brazil), 1996-2012}

Annelise C. Gonçalves 1

Maria da Conceição N. Costa 2

Florisneide Rodrigues Barreto 3

Jairnilson S. Paim 4

Estela Maria R. Nascimento 5

Enny Santos da Paixão 6

Eduardo Luiz Andrade Mota 7

1 Escola de Enfermagem. Universidade Federal do Rio Grande do Sul. Porto Alegre, RS, Brasil.

2-4,6,7 Instituto de Saúde Coletiva. Universidade Federal da Bahia. Rua Basílio da Gama. s.n. Canela. Salvador, BA, Brasil. CEP: 40 110-040. E-mail: mcncosta@ufba.br

${ }^{5}$ Secretaria de Saúde do Estado da Bahia. Salvador, BA, Brasil.

\section{Resumo}

Objetivos: analisar a tendência da mortalidade

Objectives: to investigate neonatal mortality trends, principal causes and potential risk factors, in Salvador-Bahia, 1996-2012.

Methods: a time series study was carried out using data from the Mortality and Live Births/LB Information Systems and the National Register of Health Establishments. Parameters for the trend over time of the neonatal mortality coefficient /NMC and the proportions of LBs in terms of characteristics of the mother, the newborn and health care were obtained using simple linear regression. Spearman's correlation coefficient was used to evaluate the relation between these variables.

Results: a decline of $21.2 \%$ in NMC was observed, mainly owing to premature births ( $\beta=$ $\left.0.730 ; p=0.006 ; R^{2}=0.423\right)$. This tendency was followed by the proportion of LBs among adolescent mothers and mothers with no schooling. The proportion of LBs among mothers aged $>35$ years, premature births and Caesarean births was found to be higher. The predominant cause of neonatal death was specific infection during perinatal period (13.2\%). Intra-uterine hypoxia lasphyxia during birth (8.4\%) and disorders relating to premature birth/low birth weight $(15.9 \%)$, were also found to be on the rise ( $\beta=$ $\left.1.319 ; p=0.006 ; R^{2}=0.428\right)$.

Conclusions: neonatal mortality and the prevalence of potential risk factors are on the decline in Salvador. Initiatives aiming to improve neonatal care and the living conditions of the population may be contributing to this trend.

Key words Mortality, Neonatal mortality, Risk factors neonatal, principais causas e potenciais fatores de

Métodos: estudo de série temporal tendo como fontes de dados os Sistemas de Informação sobre Mortalidade e sobre Nascidos Vivos/NV e Cadastro Nacional de Estabelecimentos de Saúde. Parâmetros da tendência temporal do coeficiente de mortalidade neonatal/CMN e da proporção de NV segundo características maternas, do recém-nascido e de atenção à saúde foram obtidos mediante Regressão Linear Simples. Coeficiente de Correlação de Spearman avaliou relação entre estas variáveis. $C M N$, principalmente devido ao componente precoce $\left(\beta=-0,730 ; p=0,006 ; R^{2}=0,423\right)$. Acompanhou esta tendência, a proporção de $N V$ de mães adolescentes $e$ sem instrução. A proporção de NV de mães com idade $>35$ anos, nascimentos prematuros e de partos cesáreos exibiram crescimento. Predominaram mortes neonatais por Infecções específicas do período perinatal $(13,2 \%)$, Hipóxia intrauterinal Asfixia ao nascer $(8,4 \%)$ e Transtornos relacionados à prematuridade/baixo peso ao nascer (15,9\%), estas últimas com tendência de crescimento $(\beta=1,319$; $p=0,006 ; R^{2}=0,428$ ).

Conclusões: a mortalidade neonatal e potenciais fatores de risco estão decrescendo em Salvador. Iniciativas voltadas para melhoria da atenção ao recém-nascido e das condições de vida da população podem estar contribuindo para esta tendência.

Palavras-chave Moralidade, Mortalidade neonatal, Fatores de risco risco, em Salvador-Bahia, 1996-2012.

Resultados: observou-se declínio de 21,2\% no 


\section{Introdução}

A mortalidade neonatal representa, atualmente, a parcela mais expressiva da mortalidade infantil, em várias regiões do mundo. Embora venha decrescendo, o declínio apresenta-se mais lento nos países onde o risco para esta mortalidade é elevado.1,2 Globalmente, entre 1990 e 2012, a redução nas taxas de mortalidade neonatal foi de $37 \%$, porém em menor proporção no sul da Ásia e África subsaariana e mais acentuada $(55,4 \%)$ na América Latina e Caribe, onde passou de 22/1000 nascidos vivos (NV) para 10/1000 NV, nesse período. ${ }^{3}$ Há também informações de que parcela significativa desses óbitos seja devida a causas evitáveis. ${ }^{1}$ Estimativas indicam que a prevenção de $70 \%$ dos óbitos neonatais resultaria em um decréscimo de $25 \%$ na mortalidade de crianças menores de cinco anos. Portanto, a redução dessas mortes representa um passo essencial para o alcance de uma das metas de desenvolvimento do milênio que prevê melhorias nos indicadores de saúde infantil e materna. ${ }^{2}$

No Brasil, a mortalidade neonatal ultrapassou a pós-neonatal no conjunto das capitais a partir de 19874 e passou a ser predominante no país como um todo em meados dos anos de 1990.5 Entre 1983-85, os óbitos neonatais representavam em média $43,7 \%$ dos óbitos de menores de um ano e $31,1 \%$ deles ocorriam no período neonatal precoce. No período 1993-1995, essas médias eram, respectivamente, de $52,9 \%$ e $41,5 \%$. As taxas de mortalidade desses componentes da mortalidade infantil eram de 19,8/1000 NV e 15,6/1000 NV em 1997 e de 14,2/1000 NV e 10,9/1000 NV em 2005.6 Em 2013, exibindo taxa de $9,2 / 1000 \mathrm{NV}$, a mortalidade neonatal constituía $68,6 \%$ dos óbitos dos menores de um ano no país. ${ }^{7}$ Este valor é aproximadamente 2,3 vezes superior à dos Estados Unidos (4/1000 NV), em 2012, e quase dez vezes maior que a apresentada pelo Japão (1/1000 NV), país que ostenta uma das menores taxas de mortalidade infantil do mundo. ${ }^{1}$ Apesar dos progressos observados no setor saúde no Brasil, 8 a evitabilidade de parcela considerável dos óbitos neonatais e sua distribuição desigual entre as regiões, ${ }^{9}$ indicam a necessidade de ações voltadas para modificar este cenário, especialmente entre grupos sociais menos favorecidos. No Estado da Bahia, situado no Nordeste (uma das regiões menos desenvolvidas do Brasil), o risco para a mortalidade neonatal em 2013 foi de 12,5/1000NV. Na sua capital, Salvador, este risco foi de 13,1/1000 NV, ou seja, aproximadamente cinco vezes superior à de Florianópolis, que apresenta uma das menores taxas de mortalidade neonatal $(2,7 / 1000 \mathrm{NV})$ entre as capitais brasileiras. ${ }^{7}$

Entende-se que estudos de séries temporais possibilitam a identificação de padrões atípicos na evolução dos níveis da morbimortalidade e na estrutura de suas causas, além de serem úteis para a avaliação do impacto produzido por intervenções. Em vista disso, e considerando que, a partir dos anos 2000, o Brasil passou a ser alvo de políticas públicas sociais e econômicas voltadas para a população de baixa renda, em especial, no campo da educação e da saúde, 10 torna-se importante verificar se essas intervenções foram acompanhadas de alguma mudança na tendência apresentada pela mortalidade neonatal.

O presente estudo teve como objetivo analisar a tendência temporal da mortalidade neonatal, de suas principais causas e de potenciais fatores de risco, em Salvador-Bahia, 1996-2012.

\section{Métodos}

Realizou-se um estudo de série temporal da mortalidade neonatal entre os nascidos vivos de mães residentes em Salvador, Bahia, de 1996 a 2012. Sistemas de Informação sobre Mortalidade (SIM) e sobre Nascidos Vivos (SINASC) foram as fontes de dados sobre óbitos e nascidos vivos, respectivamente. Também foram provenientes do SIM, os óbitos neonatais por causas evitáveis, já classificados segundo a Lista Brasileira de Causas de Mortes Evitáveis por Intervenções do Sistema Único de Saúde (SUS).11 O número de leitos hospitalares de 2000 a 2012 foi obtido do Cadastro Nacional de Estabelecimentos de Saúde/CNES/ MS.12 Aqueles dos anos de 1996 a 1999 não estavam disponíveis.

Foram calculados os coeficientes de mortalidade neonatal e de seus componentes (precoce e tardio) por 1000/NV, a proporção (em \%) dessas mortes por grupos de causas (Capítulos CID 10), por grupos de causas evitáveis e pelas três principais causas específicas (CID BR10 - categoria de três caracteres). Calculou-se ainda a proporção de nascidos vivos segundo características maternas, do recém-nascido e de atenção à saúde (nascidos vivos com peso ao nascer $<2500$ g, idade gestacional $<37$ semanas, mães de 10 a 19 anos e de 35 anos ou mais, mães sem nenhuma instrução e daquelas com menos de sete consultas de pré-natal e que tiveram partos cesáreos). Estes percentuais só incluem os nascidos vivos para os quais a informação sobre a variável de interesse era conhecida. Como o número de nascidos vivos de $<37$ semanas de gestação e de peso $<2500$ 
gramas apresentava, em 1999, valor muito baixo em relação aos dos demais anos, optou-se por utilizar, para este ano, o valor da média aritmética da proporção dos anos anterior e posterior, de cada uma dessas variáveis. Para fins de comparação, também foram calculados os coeficientes de mortalidade neonatal para o Estado da Bahia e o Brasil.

Curvas de tendência foram construídas para todos os indicadores. Mediante análise de Regressão Linear Simples obteve-se os parâmetros da tendência temporal, considerando-se nível de significância de 0,05 . Presença de autocorrelação entre os termos da regressão foi examinada pela estatística DurbinWatson e, quando esta era identificada (valor distante de 2), aplicava-se o modelo Prais-Winsten no tratamento dos resíduos autocorrelacionados. $13 \mathrm{~A}$ relação entre evolução temporal da mortalidade neonatal e de características maternas, do recémnascido e de atenção à saúde, no período 1996-2012, foi avaliada pelo teste de correlação de Spearman $(p=0,05)$.

Este estudo foi realizado a partir de dados secundários de domínio público, disponíveis no DATASUS, que não identificam os sujeitos da pesquisa. Desse modo, não é necessária a sua aprovação por um Comitê de Ética em Pesquisa.

\section{Resultados}

Do total dos óbitos de menores de um ano residentes em Salvador em 1996 e em 2012, respectivamente, $61,9 \%$ (689) e $71,1 \%$ (441) ocorreram no período neonatal. Estes valores corresponderam ao aumento de $14,9 \%$ na participação relativa desse componente e decréscimo de $36,0 \%$ no número absoluto de óbitos. Os coeficientes de mortalidade neonatal e de seus componentes precoce e tardio foram, respectivamente, de 15,9, 12,6 e 2,5/1000 NV em 1996 e de $11,9,9,1$ e 2,7/1000 NV em 2012. Entre 1998 e 2003 foram registrados os maiores valores para a mortalidade neonatal, que variaram entre $23,0 / 1000 \mathrm{NV}$, em 1999 e 17,8/1000 NV em 1998 (Figura 1). A redução deste indicador, considerando o primeiro e o último ano do período do estudo, foi de $21,2 \%$, porém o decréscimo só se tornou mais consistente a partir de 2005.

De 1996 a 2012, tanto o coeficiente de mortalidade neonatal $\left(\beta=-0,793 ; p=0,009 ; \mathrm{R}^{2}=0,394\right)$ quanto o neonatal precoce $(\beta=-0,730 ; p=0,006$; $\left.\mathrm{R}^{2}=0,423\right)$ apresentaram tendência decrescente estatisticamente significante, no período estudado. Também para o Brasil a tendência desses indicadores foi de queda estatisticamente significante, embora com menor velocidade, com redução anual de 0,187 óbitos neonatais para cada $1000 \mathrm{NV}$ e de 0,290 óbitos neonatais precoces para cada $1000 \mathrm{NV}$, no período de 1996 a 2012. Na Bahia, o declínio não mostrou significância estatística (Figura 1).

Verifica-se na Figura 2 que entre os potenciais fatores de risco analisados, a proporção de nascidos vivos de mães adolescentes em Salvador $(\beta=-0,678$; $\left.p<0,001 ; \mathrm{R}^{2}=0,827\right)$ apresentou tendência de declínio, enquanto para a proporção de mães com idade igual ou superior a 35 anos exibiu crescimento significante $\left(\beta=0,507 ; p<0,001 ; \mathrm{R}^{2}=0,984\right)$. $\mathrm{O}$ aumento da proporção de nascidos vivos cujas mães receberam menos de sete consultas pré-natais $\left(\beta=0,590 ; p=0,204 ; \mathrm{R}^{2}=0,113\right)$, assim como a redução da proporção de nascidos vivos de mães sem instrução $(\beta=-0,007 ; p=0,934 ; \mathrm{R} 2=0,000)$ não foram significantes.

A Figura 3 mostra que foi estatisticamente significante a tendência de crescimento da proporção de nascidos vivos prematuros $(\beta=0,402 ; p<0,001$; $\left.\mathrm{R}^{2}=0,640\right)$ e daqueles que nasceram de parto cesáreo $\left(\beta=1,443 ; p<0,001 ; R^{2}=0,809\right)$. Já o aumento da proporção de nascidos vivos de baixo peso $(\beta=0,025$; $p=0,417 ; \mathrm{R} 2=0,048)$, bem como o decréscimo do número de leitos obstétricos por $1000 \mathrm{NV}(\beta=-0,408$; $p=0,692 ; \mathrm{R} 2=0,016)$ não mostraram significância estatística.

Na Tabela 1, observa-se que a evolução do coeficiente de mortalidade neonatal apresentou correlação negativa com a evolução da proporção de nascidos vivos de $<37$ semanas de gestação $(r h o=-0,649$; $p=0,005$ ), partos cesarianos ( $r h o=-0,725 ; p=0,001)$, mães com idade $\geq 35$ anos $(\mathrm{rho}=-0,674 ; p=0,003) \mathrm{e}$ leitos obstétricos/1000 NV (rho= $=0,593 ; p=0,033) \mathrm{e}$ correlação positiva com a evolução da proporção de nascidos vivos de mães adolescentes $(\mathrm{rho}=0,712$; $p=0,001)$ e de mães analfabetas (rho $=0,645$; $p=0,005)$.

No que se refere às causas de mortes neonatais, em 1996, as afecções originadas no período perinatal foram responsáveis por $85,9 \%$, vindo a seguir as malformações congênitas, deformidades e anomalias cromossômicas com $11,3 \%$. Esses percentuais foram de $81,9 \%$ e $17,2 \%$, respectivamente, em 2012 . Neste último ano, 79,1\% (349) dos óbitos neonatais foram devidos a causas claramente evitáveis, das quais 89,4\% (267) reduzíveis por adequada atenção na gestação, parto e recém-nascido, assim distribuídos: $52,7 \%$ (184) evitáveis por adequada atenção à mulher na gestação, 32,7\% (114) por adequada atenção ao recém-nascido e $14,0 \%$ (49) por adequada atenção ao parto. 


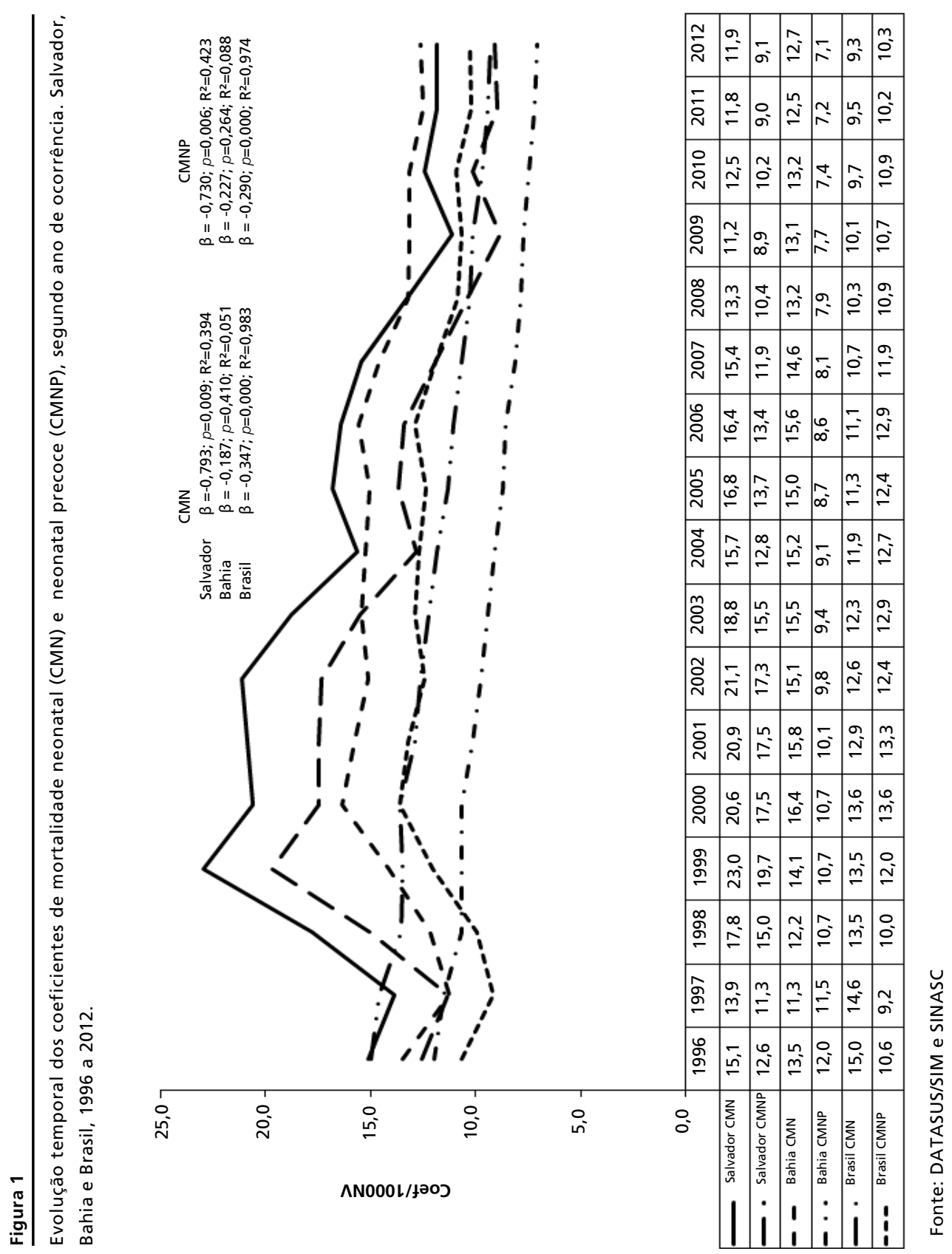




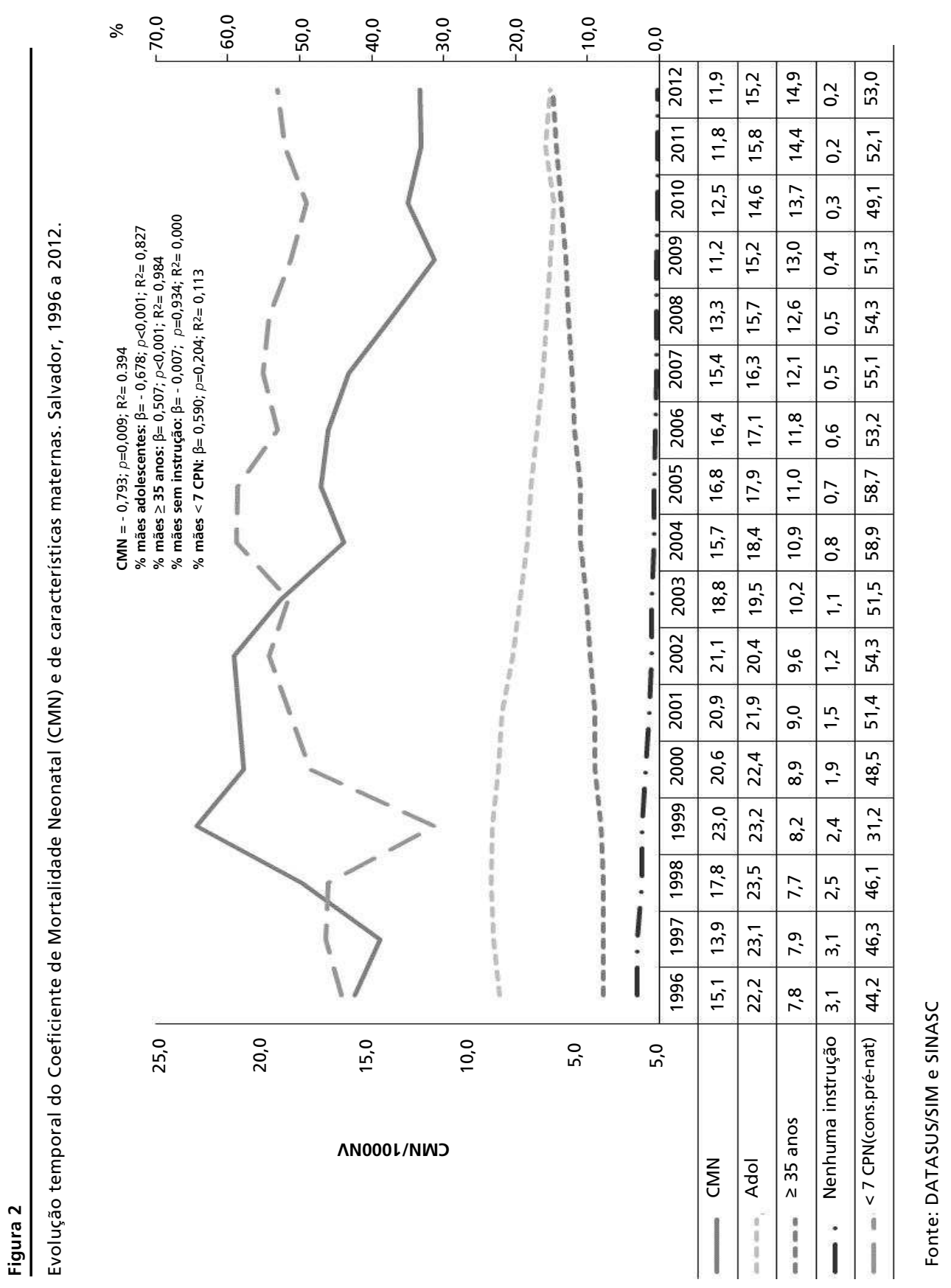




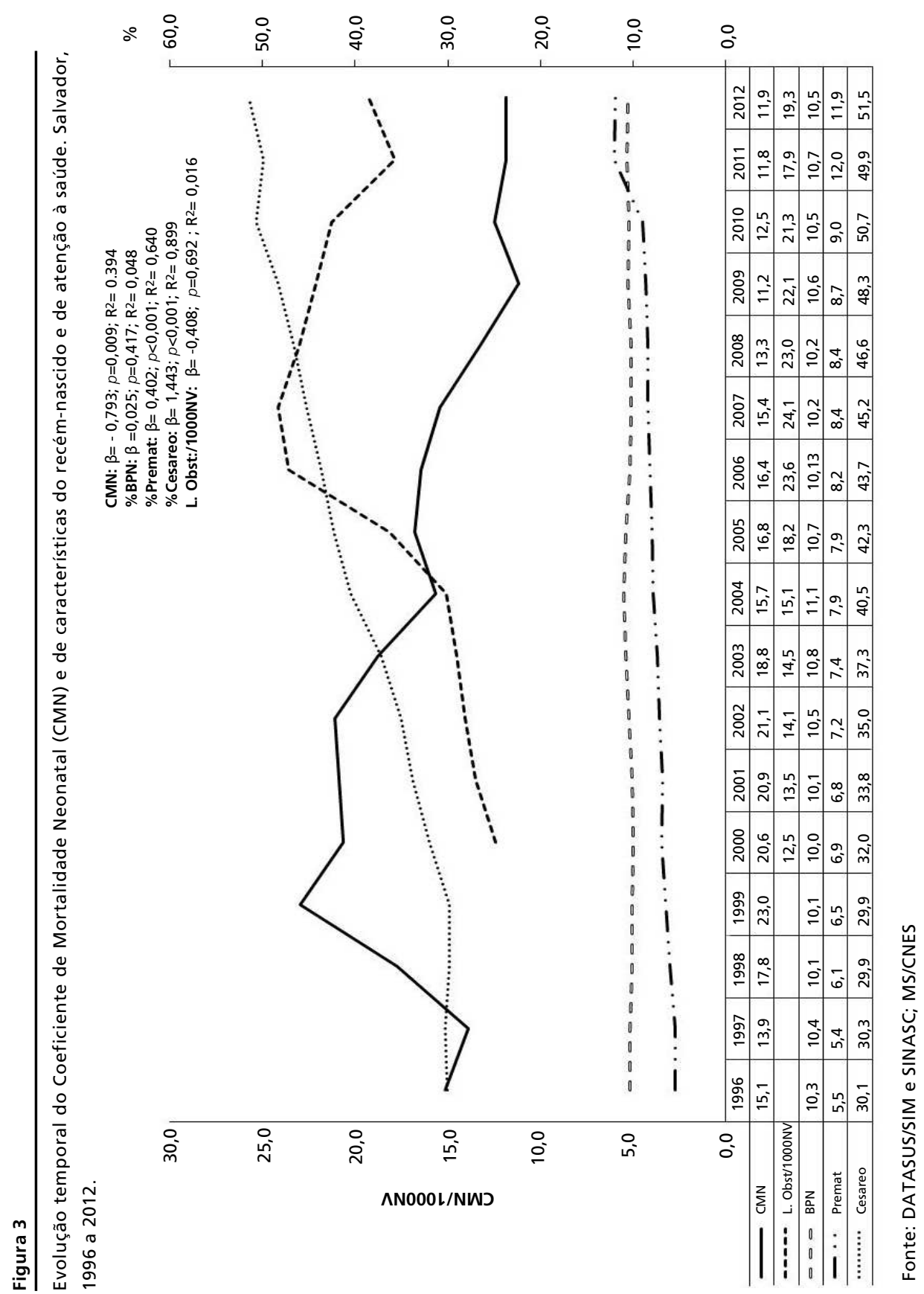




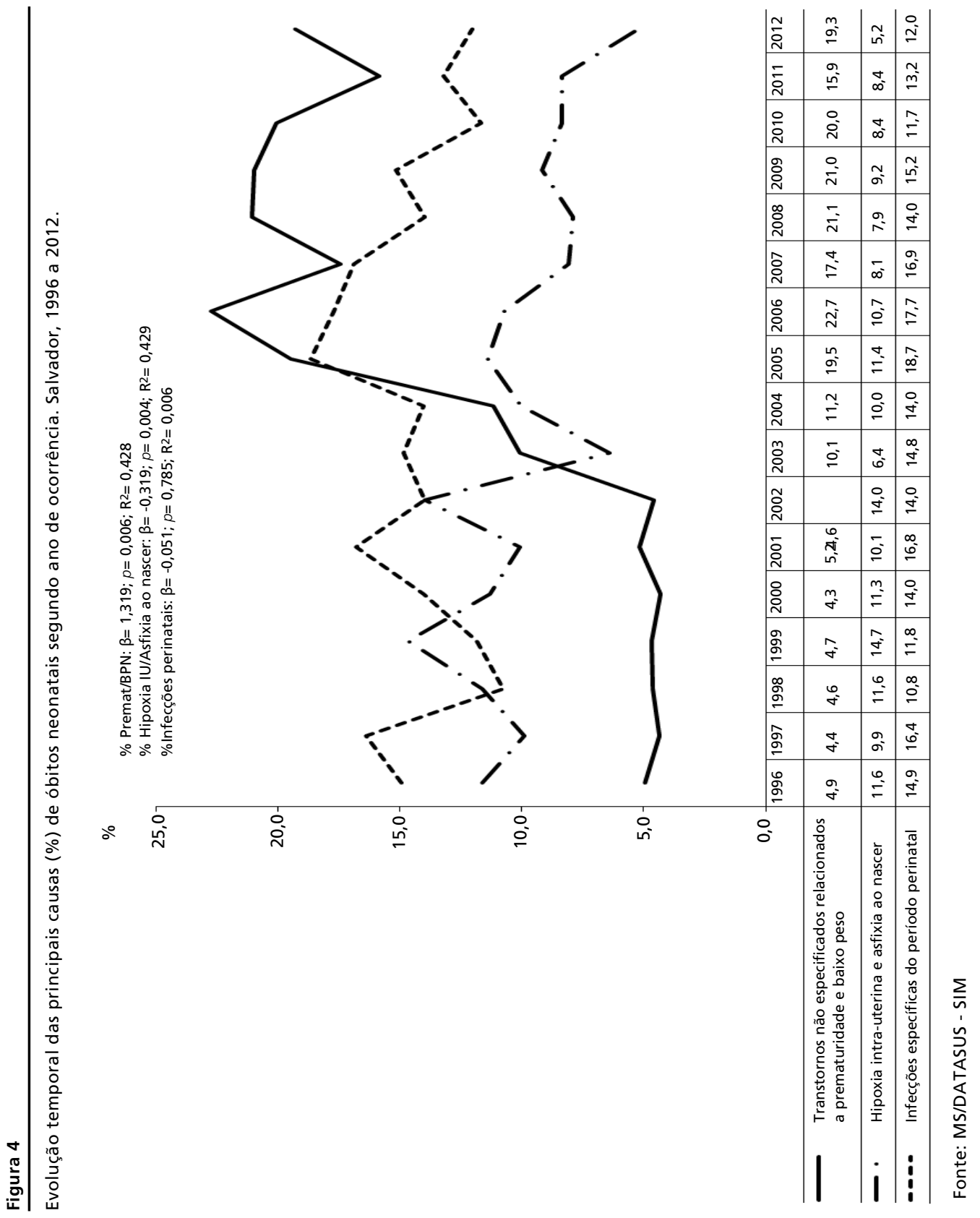


A Figura 4 apresenta as três causas específicas de óbitos neonatais mais frequentes: transtornos não específicos relacionados à prematuridade e ao baixo peso ao nascer $(15,9 \%)$ que apresenta tendência de crescimento $\left(\beta=1,319 ; p=0,006 ; \mathrm{R}^{2}=0,428\right)$, hipóxia intrauterina/asfixia ao nascer $(8,4 \%)$ com tendência de declínio $\left(\beta=-0,319 ; p=0,004 ; \mathrm{R}^{2}=0,429\right)$ e infecções específicas do período perinatal $(13,2 \%)$ cuja redução não foi estatisticamente significante $\left(\beta=-0,051 ; p=0,785 ; \mathrm{R}^{2}=0,006\right)$.

\section{Tabela 1}

Coeficiente de Correlação de Spearman (rho) para a relação entre os coeficientes* de mortalidade neonatal (CMN), neonatal precoce (CMNP) e variáveis selecionadas. Salvador, 1996-2012.

\begin{tabular}{lcccc}
\hline \multirow{2}{*}{ Variáveis } & \multicolumn{2}{c}{ CMN } & \multicolumn{2}{c}{ CMNP } \\
\cline { 2 - 5 } & rho & $p$ & rho & 0,075 \\
Baixo peso ao nascer (\%) & $-0,400$ & 0,112 & $-0,443$ & 0,003 \\
Prematuridade (\%) & $-0,649$ & 0,005 & $-0,669$ & 0,001 \\
Parto Cesáreo (\%) & $-0,725$ & 0,001 & $-0,746$ & 0,017 \\
Leitos obstétricos/1000 NV\# & $-0,593$ & 0,033 & $-0,647$ & 0,001 \\
Mães adolescentes (\%) & 0,712 & 0,001 & 0,749 & 0,002 \\
Mães com idade $\geq 35$ anos (\%) & $-0,674$ & 0,003 & $-0,695$ & 0,504 \\
$<7$ consultas pré-natais (\%) & $-0,121$ & 0,643 & $-0,174$ & 0,003 \\
Mães analfabetas (\%) & 0,645 & 0,005 & 0,668 & \\
\hline
\end{tabular}

* por 1000 NV; \# Dados a partir do ano 2000.

\section{Discussão}

A mortalidade neonatal no município de Salvador apresentou tendência decrescente, entre os anos de 1996 e 2012, a exemplo do que vem ocorrendo em Guarulhos-SP.14 A despeito desta evolução favorável, a magnitude de sua ocorrência naquela capital, em 2012, ainda era cerca de três vezes superior ao de países desenvolvidos. ${ }^{1}$ Tal situação evidencia que segue sendo necessária a intensificação de investimentos em saúde voltados à gestação, parto e nascimento, nesta capital.

Conforme já demonstrado em outras investigações, 15,16 várias características maternas, assistenciais e dos recém-nascidos representam importantes fatores de risco para a mortalidade neonatal. Assim como observado no presente estudo, algumas delas vem exibindo tendência de declínio também no Brasil, como a redução de nascidos vivos de mães adolescentes, enquanto outras vem aumentando como o nascimento de crianças prematuras, de mães com idade $\geq 35$ anos e de partos cesáreos. ${ }^{10,17}$ Nesse particular, apesar de não indicar causalidade, vale destacar a significante associação positiva apresentada, em Salvador, entre a mortalidade neonatal e nascidos vivos de mães adolescentes, bem como a associação inversa entre este indicador e prematuri- dade, partos cesáreos e mães com 35 anos e mais.

A redução de nascidos vivos de mães adolescentes é um achado positivo, visto que a gravidez nesta fase da vida geralmente está associada a desfechos desfavoráveis. 18 Por sua vez, conquanto sejam preocupantes o crescimento de nascidos vivos de mulheres com idade igual ou superior a 35 anos, assim como daqueles por cesariana, por poderem elevar o risco de nascimentos prematuros e de morte neonatal, 19,20 com relação à cesariana, sabe-se que este tipo de parto também pode ter efeito protetor para esta mortalidade, ${ }^{21}$ se indicado adequadamente. Portanto, não surpreende a relação inversa observada nesse estudo, fato também constatado em estudo multicêntrico abrangendo Unidades Neonatais de Alto Risco Nordeste do país. ${ }^{22}$ Entretanto, caberia investigar as condições socioeconômicas das gestantes submetidas a este tipo de parto, pois na hipótese de a maioria delas possuir melhor condição de vida e saúde, possivelmente, teria maiores chances para realizar o parto com profissionais qualificados e com estrutura e tecnologia adequadas, de modo que, consequentemente, seus recém-nascidos apresentariam maior chance de sobrevivência. Estes fatores também poderiam explicar a relação inversa encontrada entre a mortalidade neonatal e mães de $\geq 35$ anos de idade, na medida em que é mais 
provável que sejam as mulheres de melhores condições de vida que retardam a maternidade para após sua realização profissional. Este fato foi observado no estudo realizado em Porto Alegre (RS) que encontrou maior proporção de partos operatórios e com idade igual ou maior a 35 anos no grupo de mães com maior nível de escolaridade. 23

Em Salvador, no período de 2007 a 2012, a cobertura de leitos de Unidade de Tratamento Intensivo Neonatal (UTIN) era de 3,5/1000 NV12 e, portanto, superior ao parâmetro $(2 / 1000 \mathrm{NV}) \mathrm{de}$ necessidade populacional estabelecido pelo Ministério da Saúde nas Diretrizes para Organização da Atenção a Recém-nascidos de risco. ${ }^{24}$ Uma vez que esta capital concentra cerca de $70 \%$ desses leitos, possivelmente, a elevada cobertura de UTIN pode ser uma das hipóteses explicativas para a correlação inversa encontrada neste estudo entre a mortalidade neonatal e os nascimentos prematuros, embora tal relação não indique causalidade. Por outro lado, esta elevada concentração de leitos de UTIN em apenas um município, pode ser um dos fatores que estão determinando a evolução desfavorável da mortalidade neonatal no Estado da Bahia como um todo, ainda que a capital deva atender a uma parcela da demanda de outros municípios por este tipo de leito. A baixa velocidade do declínio desta mortalidade no Brasil, já que representa a média nacional, pode ser devida a influência das regiões mais carentes, como o Norte e Nordeste do país, que vem apresentando persistentemente elevadas taxas de mortalidade infantil. 5,10

A não significância estatística da tendência de crescimento do baixo peso ao nascer talvez tenha resultado do fato desta variável não ter sido ajustada para a idade gestacional, visto que vem se observando aumento deste fator acompanhando a tendência de crescimento dos nascimentos prétermo. 23

O predomínio de causas de mortes relacionadas às afecções originadas no período perinatal e de seus três tipos mais frequentes é similar à situação encontrada em quase todo o mundo, 25 e também em outros municípios do Brasil.16,26 Porém, chama a atenção a grande magnitude com que essas mortes ocorrem em Salvador e o fato de cerca de $80 \%$ delas serem evitáveis, na grande maioria, por adequada atenção à gestação, ao parto e ao recém-nascido. Isto depõe contra a qualidade da atenção à saúde oferecida às gestantes e indica a possibilidade de deficiência na resolutividade da assistência pré-natal. Com relação ao aumento da participação das anomalias congênitas, que passaram a ocupar a segunda posição entre os principais grupos de causas de mortes neonatais em Salvador, é possível que esteja refletindo uma melhoria do diagnóstico nos serviços públicos de saúde. 27

Embora, o nascimento pré-termo, infecções e hipóxia/asfixia ao nascer sejam, mundialmente, as principais causas de morte neonatal, ${ }^{3}$ sua magnitude varia entre países, sendo mais elevada naqueles menos desenvolvidos. No Brasil, estas causas estão relacionadas ao manejo do parto e hipóxia/asfixia ao nascer, que apresentam grande potencial de prevenção, vez que $98 \%$ dos partos nesse país ocorrem em maternidades e $97 \%$ são atendidos por médicos ou enfermeiros. ${ }^{8}$ Em Salvador, dados de 2013 indicam que praticamente $100 \%$ dos nascimentos ocorreram em hospitais, sob os cuidados de profissionais de saúde.7 Quanto às mortes neonatais por infecções de origem perinatal, é possível que estejam refletindo o baixo nível de saúde das gestantes que chegam para o parto trazendo infecções não diagnosticadas ou tratadas inadequadamente no pré-natal. Também o manejo inapropriado do parto e do recém-nascido, as internações prolongadas e a insuficiência de materiais e equipamentos em hospitais podem expor gestantes, parturientes e recém-nascidos ao risco de infecções. 28

As causas não especificadas relacionadas à prematuridade e ao baixo peso ao nascer, principais causas de mortes neonatais em Salvador desde 2005, chamam atenção pela sua relação com a baixa qualidade da atenção dada às gestantes, durante o prénatal, especialmente no que se refere à abordagem e manejo adequado de fatores de risco para sua ocorrência, 16,20,25 embora também deva ser considerada a participação dos partos cesáreos na determinação do baixo peso ao nascer e prematuridade, 19,20 conforme já referido.

Dados de pesquisa multicêntrica realizada no Brasil indicaram que, em 2011-2012, também a prematuridade e suas complicações eram importantes causas evitáveis de óbito neonatal. ${ }^{29}$ É sabido que mesmo em países desenvolvidos com amplo acesso a serviços de saúde essas causas ainda não foram eliminadas 25 devido, possivelmente, à complexidade dos mecanismos biológicos e de sua interação com fatores sociais, econômicos e comportamentais de difícil controle. Apesar disso, observase que, em Salvador, ainda é possível reduzir em muito a sua prevalência em razão dos altos níveis de sua ocorrência. Inciativas como o "Programa de Humanização do Pré-natal e Nascimento" (PHPN), "Programa de Gravidez de Alto Risco", criação das "Redes de Perinatologia" e, mais recentemente (2011), a "Estratégia Rede Cegonha" ainda não conseguiram um impacto consistente na mortalidade 
neonatal no país, haja vista que a prematuridade se mantém entre as principais causas de óbitos neonatais. 6,29

Salienta-se que limitações inerentes à utilização de dados secundários, como o preenchimento incorreto e incompleto de alguns campos da Declaração de Óbitos e/ou erro na classificação óbitos como fetal ou não fetal 30 impõem cautela quando da interpretação dos resultados deste estudo. Presença de valores discrepantes de alguma variável pode ter afetado a sua tendência. Além disso, a grande flutuação no número de óbitos neonatais entre 1996 e 2003 é indicativa da existência de alguma fragilidade do SIM na captação desses óbitos, naquele período,

\section{Referências}

1. WHO (World Health Organization). World Health Statistics 2014. Geneva; 2014. [acesso em 27 jul 2015]. Disponível em: http://www.who.int/gho/publications/world_health statistics/EN_WHS2014_Full.pdf.

2. Rajaratnam JK, Marcus JR, Flaxman AD, Wang H, LevinRector A, Dwyer L, Costa M, Lopez AD, Murray CJ Neonatal, postneonatal, childhood, and under-5 mortality for 187 countries, 1970-2010: a systematic analysis of progress towards Millennium Development Goal 4. Lancet. 2010; 375 (9730): 1988-2008.

3. UNICEF (United Nations Children's Fund). Committing to Child Survival: a Promise Renewed. Progress Report 2013, september 2013. [acesso em 27 jul 2015]. Disponível em: http://www.unicef.org/lac/Committing_to_Child_Survival_ APR_9_Sept_2013.pdf

4. Costa MCN, Mota ELA, Paim JS, Vieira da Silva LM, Teixeira MG, Mendes CMC. Mortalidade infantil no Brasil em períodos recentes de crise econômica. Rev Saúde Pública. 2003; 37 (6): 699-706.

5. Brasil. Ministério da Saúde. Secretaria de Vigilância em Saúde. Departamento de Análise de Situação de Saúde. Saúde Brasil 2009: uma análise da situação de saúde e da agenda nacional e internacional de prioridades em saúde. Brasília, DF; 2010.

6. França E, Lansky S. Mortalidade infantil neonatal no Brasil: situação, tendências e perspectivas. Rede Interagencial de Informações para Saúde, organizador. Demografia e saúde: contribuição para análise de situação e tendências. Brasília, DF: Organização Pan-Americana da Saúde; 2009.p. 83-112.

7. Brasil. Ministério da Saúde. Indicadores e Dados Básicos Brasil 2013. [acesso em 27 jul 2015]. Disponível em: http://w3. datasus. gov. br/datasus/datasus.php

8. Brasil. Ministério da Saúde. Pesquisa Nacional de Demografia e Saúde da Criança e da Mulher - PNDS 2006: dimensões do processo reprodutivo e da saúde da criança/ Ministério da Saúde, Centro Brasileiro de Análise e Planejamento. Brasília, DF; 2009. em Salvador. Entretanto, a cobertura dos Sistemas de Informação sobre Mortalidade/SIM e sobre Nascidos Vivos/Sinasc em Salvador já é superior a $90 \%$ e, à exceção do número de consulta pré-natal de 1996 a 1999 e da duração da gestação em 2011, em geral, o percentual de não registro da informação sobre as variáveis obtidas do SINASC foi baixo e decrescente, sendo na maioria inferior a $1 \% .{ }^{11}$

Assim sendo, pode-se concluir que ainda se fazem necessárias políticas públicas sociais e de saúde voltadas para a melhoria das condições de vida e maior qualificação dos serviços de atenção à saúde materno-infantil com vistas intensificar a tendência declinante da mortalidade neonatal nesta capital.
9. Oliveira RRC, Silva DKF, Bonfim C. Mortalidade neonatal e evitabilidade: uma análise do perfil epidemiológico. Rev Enferm UERJ. 2011; 19 (1): 114-20. 10. Victora CG, Aquino EM, Leal MC, Monteiro CA, Barros FC, Szwarcwald CL. Maternal and child health in Brazil: progress and challenges. Lancet. 2011; 377: 1863-76.

11. Brasil. Ministério da Saúde. Departamento de Informática do SUS/Datasus. Informações de Saúde (TABNET). Estatísticas Vitais. [acesso em 18 jan 2014]. Disponível em: http://www2.datasus.gov.br/DATASUS/index.php?area=02 05 .

12. Brasil. Ministério da Saúde. Secretaria de Atenção à Saúde. Cadastro Nacional de Estabelecimentos de Saúde (CNES). [acesso em 27 jul 2015]. Disponível em: http://tabnet.datasus.gov.br/cgi/tabcgi.exe?cnes/cnv/leiutib a.def

13. Bruin J. Newtest: command to compute new test. UCLA: Academic Technology Services, Statistical Consulting Group. SAS FAQ: How can I compute Durbin-Watson statistic and 1st order autocorrelation in time series data? Available from: http://www.ats.ucla.edu/stat/sas/faq/autocorrelation_1st_order_reg.htm

14. Bando DH, Kawano MK, Kumagai LT, Gouveia JLV, Reis TM, Bernardo ES. Tendência das taxas de mortalidade infantil e de seus componentes em Guarulhos-SP, no período de 1996 a 2011. Epidemiol Serv Saúde. 2014; 23 (4): 767-72.

15. Kassar SB, Melo AMC, Coutinho SB, Lima MC, Lira PIC. Determinants of neonatal death with emphasis on health care during pregnancy, childbirth and reproductive history. J Pediatr. 2013; 89 (3): 269-77.

16. Nascimento RM, Leite AJM, Almeida NMGS, Almeida PC, Silva CF. Determinantes da mortalidade neonatal: estudo caso-controle em Fortaleza, Ceará, Brasil. Cad Saúde Pública. 2012; 28 (3): 559-72.

17. Rozario S, Brito AS, Kale PL, Fonseca SC. Série temporal de características maternas e de nascidos vivos em Niterói, RJ. Rev Bras Saúde Matern Infant. 2013; 13 (2): 137-46. 
18. Oliveira EFV, Gama SGN, Silva CMFP. Gravidez na adolescência e outros fatores de risco para mortalidade fetal e infantil no município do Rio de Janeiro, Brasil. Cad Saúde Pública. 2010; 26: 576-8.

19. Villar J, Valladares E, Wojdyla D, Zavaleta N, Carroli G, Velazco A, Shah A, Campodónico L, Bataglia V, Faundes A, Langer A, Narváez A, Donner A, Romero M, Reynoso S, Pádua KS, Giordano D, Kublickas M, Acosta A; WHO 2005 global survey on maternal and perinatal health research group. Caesarean delivery rates and pregnancy outcomes: the 2005 WHO global survey on maternal and perinatal health in Latin America. Lancet. 2006; 367: 181929.

20. Vanderlei LCM, Simões FTPA, Vidal SA, Frias PG Avaliação de preditores do óbito neonatal em uma série histórica de nascidos vivos no Nordeste brasileiro. Rev Bras Saúde Matern Infant. 2010, 10 (4): 449-58.

21. Zanini RR, Moraes AB, Giugliani ERJ, Riboldi J. Determinantes contextuais da mortalidade neonatal no Rio Grande do Sul por dois modelos de análise. Rev Saúde Pública. 2011; 45: 79-89

22. Silva CF, Leite AJM, Almeida NMGS, Ponce de Leon ACM, Olofin I, Rede Norte-Nordeste de Saúde Perinatal. Fatores associados ao óbito neonatal de recém- nascidos de alto risco: estudo multicêntrico em Unidades Neonatais de Alto Risco no Nordeste brasileiro. Cad Saúde Pública. 2014; 30 (2): 355-68.

23. Hernandez AR, Silva CH, Agranonik M, Quadros FM, Goldani MZ. Tendências das taxas de mortalidade infantil e de seus fatores de risco na cidade de Porto Alegre, Rio Grande do Sul, Brasil, no período de 1996 a 2008. Cad Saúde Pública. 2011; 27: 2188-96.
24. Brasil. Ministério da Saúde. Gabinete do Ministro. Portaria $\mathrm{N}^{\circ}$ 930, de 10 de maio de 2012. Define as Diretrizes e Objetivos para a organização da atenção integral e humanizada ao recém-nascido grave ou potencialmente grave e os critérios de classificação e habilitação de leitos de Unidade Neonatal no âmbito do Sistema Único de Saúde (SUS). Disponível em: http://bvsms.saude.gov.br/bvs/saudelegis/ gm/2012/prt0930_10_05_2012.html

25. Lawn JE, Cousen S, Zupan J. 4 million neonatal deaths: when? Where? Why? Lancet. 2005; 365 (9462): 891-900.

26. Maia LTS, Souza WV, Mendes ACG. Diferenciais nos fatores de risco para a mortalidade infantil em cinco cidades brasileiras: um estudo de caso-controle com base no SIM e no SINASC. Cad Saúde Pública. 2012; 28 (11): 2163-76.

27. Bahia. Secretaria Estadual de Saúde. Superintendência de Vigilância em Saúde. Diretoria de Informação em Saúde: Mortalidade Infantil no estado da Bahia: Situação atual e perspectivas. Disponível em: http://www1.saude.ba.gov.br/dis/arquivos_pdf/Mort_Infant EstadoBa.pdf. Acessado em 21/08/2015

28. Mussi-Pinhata MM, Nascimento SD. Infecções neonatais hospitalares. J Pediatr (Rio J.). 2001; 77 (Supl. 1): S81-96.

29. Lansky S, Friche AAL, Silva AAM, Campos D, Bittencourt DAS, Carvalho ML, Frias PG, Cavalcante RS, Cunha AJLA. Pesquisa Nascer no Brasil: perfil da mortalidade neonatal e avaliação da assistência à gestante e ao recémnascido. Cad Saúde Pública. 2014; 30 (Supl.): S192-207.

30. Mascarenhas MDM, Gomes KRO. Confiabilidade dos dados do Sistema de Informações sobre Nascidos Vivos em Teresina, Estado do Piauí, Brasil - 2002. Ciênc Saúde Coletiva. 2011; 16 (Suppl.1): 1233-9.
Recebido em 25 de maio de 2015

Versão final apresentada em 1 de julho de 2015

Aprovado em 10 de julho de 2015 